\title{
Evaluation de l'efficacité externe de la formation des agriculteurs
}

\author{
Alain Mingat et Nelly Stephan \\ IREDU-CNRS et Université de Bourgogne ; ENESAD
}

Cette recherche a reçu une aide financière de la part de la DEP du Ministère de l'Education Nationale

(comité "investissement éducatif et son efficacité" ; convention nº 92-196).

Depuis une vingtaine d'années, les pouvoirs publics, dans le secteur agricole, associent politique de formation et politique d'installation. Ceci se traduit par la volonté d'imposer des minima de formation pour les agriculteurs qui désirent s'installer et bénéficier des aides de l'Etat (prêts bonifiés et dotation d'installation aux jeunes agriculteurs, DJA) en application de la directive 797 de la CEE de 1972. L'idée de base est que des agriculteurs mieux formés seront plus productifs.

Si, dans les années 70, le futur agriculteur devait seulement suivre un stage de formation d'adultes de 200 heures, depuis janvier 1992, ce dernier doit pour obtenir la capacité professionnelle agricole lui donnant droit à ces aides, être titulaire du Brevet de Technicien Agricole (BTA ; diplôme de niveau IV) ou de son équivalent le Brevet Professionnel de responsable d'exploitation agricole (BP REA). Chacun de ces diplômes nécessite au moins 1200 heures de formation ; le futur agriculteur doit en outre suivre un stage d'application d'une durée minimale de six mois hors de l'exploitation familiale.

Pourtant, cette décision d'exiger de tous les futurs agriculteurs français une formation qui serait au minimum égale au "niveau IV" soulève deux types de questions:

i) la première est d'ordre pratique ; en effet, un changement de niveau dans la formation agricole exigée risque de poser des problèmes à certains jeunes qui se destinent à ce métier. Ainsi, en 1991, parmi les agriculteurs ayant bénéficié de la DJA, ils n'étaient que $21,5 \%$ à justifier d'un diplôme au moins équivalent au BTA, et la progression de ce taux sur une période récente n'est que de 1 à $2 \%$ par an. Cette exigence de niveau pourrait aller à l'encontre des orientations des pouvoirs publics qui souhaitent promouvoir pour les années à venir un flux annuel moyen d'environ 10000 installations ; ii) la seconde concerne la justification même de la mesure ; en effet, si les différentes réglementations mises en place sont implicitement fondées sur l'idée d'une relation générale positive entre capital humain et efficacité économique, il n'y a en fait pas de résultats empiriques susceptibles d'éclairer la situation française quant à cette relation dans le secteur de l'agriculture. Le recours à l'évidence, qui semble avoir caractérisé la réflexion politique sur le sujet, reste un argument tout de même un peu insuffisant, compte tenu notamment du volume des ressources mobilisées pour la formation; il est donc opportun de se demander dans quelle mesure celle-ci contribue effectivement à produire les effets supposés.

Les recherches sur cette question ont pour la plupart été conduites sur des pays en voie de développement; elles ont cherché à vérifier si le niveau de qualification des agriculteurs a un impact sur le degré d'efficacité des exploitations agricoles. Les estimations ont souvent trouvé un lien positif et significatif entre l'éducation et des critères soit d'efficacité technique (niveau de la production pour un niveau donné d'emploi des facteurs) soit d'efficacité allocative (combinaison des facteurs à moindre coût). Un supplément d'instruction apparaît, dans ces recherches, lié à un accroissement de la production agricole et/ou à une amélioration de la combinaison productive. Cela dit, la transférabilité des conclusions de ces études à la France semble limitée. En effet, la spécification de la variable éducation dans la littérature est limitée à l'enseignement primaire sachant en outre que le contexte économique de ces pays diffère aussi de façon forte par rapport à celui de la France.

Les initiatives politiques concernant la formation des agriculteurs ont pour objectif premier de les rendre plus efficaces. Dans une logique de production, être efficace pour l'agriculteur, c'est d'abord savoir choisir les spéculations les plus rentables (mobilité des facteurs entre productions alternatives), c'est ensuite savoir identifier les 
modes de production les plus appropriés compte tenu de l'état de la technologie et c'est enfin faire fonctionner cette exploitation de la meilleure façon (choix des productions et des modes de production/commercialisation). Comme la question est de déterminer si les agriculteurs plus formés sont plus efficaces que les non formés, il importe donc d'examiner non seulement s'ils sont globalement plus performants, mais aussi d'identifier et d'articuler l'influence de la formation dans les domaines cités précédemment.

On peut représenter le cadre d'analyse par un schéma du type ci-après. Pour des raisons de simplicité, seules deux étapes sont ici envisagées pour identifier les effets de la formation reçue par l'exploitant. La première (flèche 1 sur le schéma) concerne l'influence de la formation sur la définition de l'exploitation (les spéculations choisies, la surface concernée, le capital physique et le personnel mobilisés). La seconde (flèche 2) concerne l'influence de la formation de l'agriculteur sur les résultats économiques de l'exploitation compte tenu de ses caractéristiques ; la flèche 2 identifie donc l'influence de la formation de l'exploitant sur sa capacité à tirer profit de son exploitation compte tenu des caractéristiques de celles-ci. La flèche 3 sur le schéma indique que de façon générale, la rentabilité économique d'une exploitation agricole est bien évidemment dépendante de ses caractéristiques distinctives (600 ha de céréales dans la Brie sont ainsi vraisemblablement associés à des résultats économiques plus élevés que 40 ha de polyculture en Auvergne).
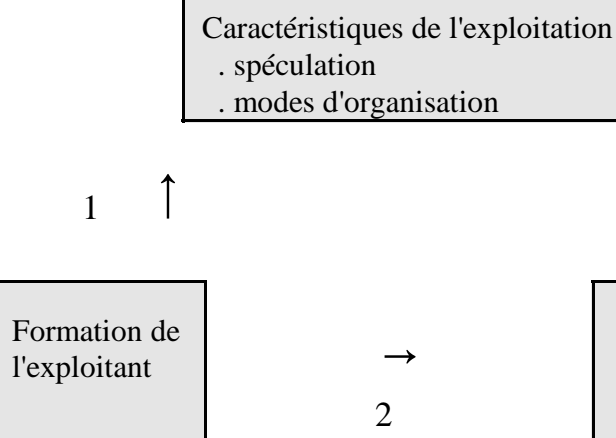

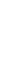

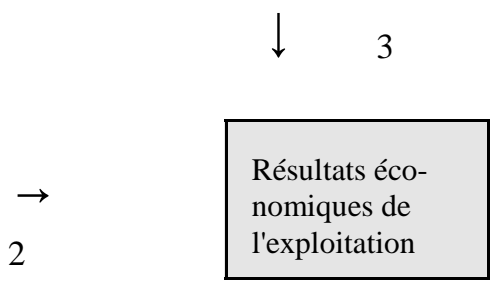

En raison du nombre de variables et de la complexité des relations concernées, et du fait que les acteurs ne sont pas distanciés par rapport aux pratiques quotidiennes, il n'est pas pertinent de se fier à leurs impressions. Plutôt que de recourir à des opinions peu fiables, nous avons mis en oeuvre une démarche de recherche fondée sur i) un regard externe sur le fonctionnement du phénomène étudié, ii) l'utilisation d'une approche comparative et iii) le recours à des méthodes quantitatives et économétriques permettant de mesurer l'intensité des relations et d'estimer l'impact des différents facteurs qui contribuent à les structurer. Nous utilisons une enquête concernant près de 1800 exploitations bourguignonnes adhérentes à un centre de gestion (données économiques) pour lesquelles les données sur l'exploitant (classe d'âge 30-50 ans) et sa formation ont été collectées par ailleurs. Nous examinons tout d'abord l'influence de la formation des chefs d'exploitation sur le choix des conditions d'exploitation, pour aborder ensuite son impact sur la capacité de l'exploitant à gérer cette exploitation.

\section{Les agriculteurs plus formés choisissent-ils une exploi- tation de caractéristiques économiques plus favora- bles?}

Il convient tout d'abord de ne pas donner un sens trop littéral au terme de choix ; en effet, on s'intéresse aux caractéristiques de l'exploitation en faisant comme si il s'agissait d'un "choix" positif fait par chaque agriculteur. La réalité est évidemment moins nette et il ne s'agit parfois pas d'un choix réel compte tenu des contraintes ou des circonstances familiales particulières des conditions d'entrée dans la profession. Cela dit, les possibilités de choix (de type de culture ou d'activités, de surface, de quantité de travail, de volume de capital) sont souvent plus importantes qu'il ne pourrait y paraître spontanément ; les changements observés sur une dizaine d'années dans le paysage agricole constituent en fait une illustration des mobilités et donc des choix possibles.

Il convient aussi de donner un contenu objectivé à la notion de "caractéristiques économiquement favorables d'une exploitation" ; ceci suppose un travail instrumental préalable. La démarche suivie ici a consisté à partir de l'idée qu'une exploitation a d'une part des caractéristiques physiques identifiables et d'autre part un résultat économique observable ; cela dit, on peut considérer que le résultat économique effectivement observé d'une exploitation a deux composantes :

i) le résultat obtenu en moyenne par les exploitations agricoles de caractéristiques physiques comparables; nous le nommerons le "résultat attendu" ;

ii) l'écart entre le résultat attendu de l'exploitation existante et le résultat effectivement observé.

Alors que la première composante est attachée à l'exploitation et à ses caractéristiques physiques, la seconde, dont la valeur moyenne est nulle par définition, est supposée être attachée à l'exploitant et à sa capacité à faire fonctionner son exploitation avec profit. Parmi les caractéristiques de l'exploitation, nous avons considéré le système de production (orientation technico-économique de l'exploitation OTEX) ${ }^{1}$, la superficie agricole utilisée

\footnotetext{
${ }^{1}$. Les productions sont ventilées dans sept orientations technicoéconomiques d'exploitation (OTEX) de la façon suivante:OTEX1: grandes cultures; OTEX2: horticulture; OTEX3: viticulture et petits fruits; OTEX4: élevages et cultures fourragères; OTEX5: élevage spécialisé; OTEX6: polyculture; OTEX7: polyculture-élevage.
} 
(SAU), le nombre d'unités de travail humain (UTH) mobilisé sur l'exploitation et le montant des investissements $^{2}(\mathrm{INV})$;

Compte tenu de la grande diversité des cas observés, il est apparu difficile de résumer le niveau de formation des agriculteurs par une variable unique type "durée des études" ; nous avons préféré utiliser plusieurs formulations : repérage des diplômes et types de formation, identification de parcours-type de formation et séparation des formations générale et agricole initiale avec prise en compte de leur degré de continuité. Concernant la rentabilité de l'exploitation agricole, il n'existe pas non plus de critère unique qui permettrait de définir, de façon complète et précise, le résultat économique d'une exploitation. En conséquence, nous avons choisi plusieurs critères empruntés à la comptabilité agricole pour comparer les résultats techniques, économiques et financiers des différentes entreprises agricoles de l'échantillon : nous avons retenu la marge brute d'exploitation et l'excédent brut d'exploitation.

La figure ci-après illustre les résultats obtenus dans l'explication de la marge brute globale (MBG) attendue sachant que la configuration (la position relative des différents parcours de formation ${ }^{3}$ ) obtenue sur la base de l'excédent brut d'exploitation attendu est en fait très comparable, laissant ainsi présager une bonne fiabilité des résultats obtenus.

On remarque que s'il existe bien une très forte variabilité du résultat économique attendu d'une exploitation à l'autre, cette variabilité ne s'explique que de façon très faible $\left(\mathrm{R}^{2}<0,05\right)$ par la formation de l'agriculteur en charge de l'exploitation. Elle s'explique par contre de façon substantielle par ses caractéristiques physiques $\left(\mathrm{R}^{2}\right.$ $=0,60)$ en dépit des imperfections dans les variables utilisées et de la simplicité de la spécification retenue.

\footnotetext{
${ }^{2}$. Le montant des investissements est, dans cette recherche, estiméà partir de la différence entre le résultat courant et l'excédent brut d'exploitation. Bien que ce critère ne soit pas parfait, il permet dans une large mesure de quantifier les investissements réalisés par l'exploitant

3 Parcours1:formationgénérale $<=$ au secondaire court, sans formation agricole initiale ou continue

Parcours 2: formationgénérale «=au secondaire court, sans formation agricole initiale avec formation agricole continue

Parcours 3 : formation générale $<=a u$ secondaire court, formation agricole initiale CAPA, avec formation agricole continue

Parcours 4 : formation générale <=au secondaire court, formation agricole initiale CAPA, sans formation agricole continue

Parcours 5 : formation générale < =au secondaire court, formation agricole initiale BEPA, avec formation agricole continue

Parcours 6 : formation générale < =au secondaire court, formation agricole initiale BEPA, sans formation agricole continue

Parcours 7: formation générale $=$ au secondaire court, formation agricole initiale BTA ou plus, avec ou sans formation agricole continue

Parcours 8 : formation générale >au secondaire court, avec ou sans formation agricole initiale, avec ou sans formation agricole continue
}

\section{Parcours de formation de l'agriculteur et résultat économique anticipé de son exploitation}

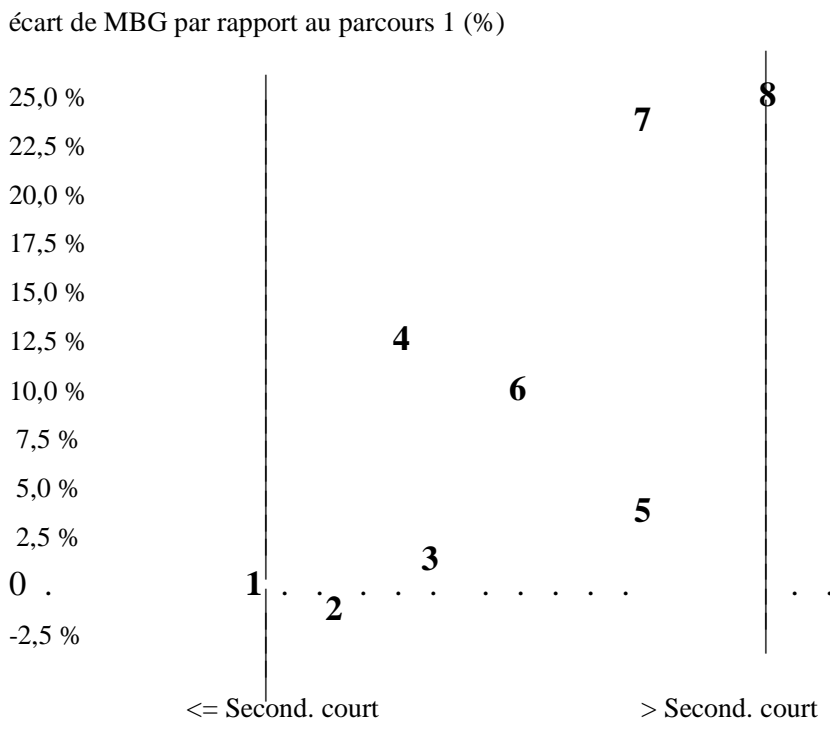

Trois observations principales peuvent être faites :

. la première est qu'il n'y a pas de différences statistiquement significatives dans le résultat économique attendu entre les parcours 1 et 2 , pour lesquels les agriculteurs ont en commun un niveau d'études générales inférieur au brevet et de ne pas avoir bénéficié de formation agricole initiale; ils différent toutefois selon la formation agricole continue : les agriculteurs ayant un parcours de formation 1 n'ont pas reçu de formation agricole initiale ni de formation agricole continue, alors que ceux qui ont un parcours 2 en ont bénéficié. Sur cette base, la formation agricole continue n'apporte donc pas de bénéfice à l'exploitant.

. la seconde observation concerne la comparaison par paires respectivement des parcours 4 et 3 et des parcours 6 et 5 . Alors que les agriculteurs ayant eu un parcours 4 ou 6 n'ont pas reçu de formation agricole continue, ceux ayant eu un parcours 3 ou 5 en ont reçue. On observe que de façon systématique et statistiquement significative, les agriculteurs qui ont reçu une formation agricole continue exploitent des structures productives dont le résultat économique attendu est inférieur à celui de leurs homologues qui n'ont pas reçu ce genre de formation (respectivement de $-12,2 \%$ et de $-6,8 \%$, ce qui est tout à fait appréciable). Il peut bien sûr éventuellement exister une sélection négative des agriculteurs qui ont suivi une formation agricole continue; cela dit, le caractère négatif apparaît suffisamment net pour qu'on puisse suggérer qu'il y a une faible pertinence de la formation agricole continue, au moins telle qu'elle a été organisée pour les agriculteurs de l'échantillon.

la troisième observation concerne cette fois les parcours 7 (agriculteurs ayant le brevet + au moins le BTA avec ou sans formation agricole continue) et 8 (agriculteurs ayant fait des études générales de niveau au moins 
égal au baccalauréat avec ou sans formation agricole initiale ou continue). On observe que ces agriculteurs (ceux ayant eu un parcours 7 de formation et plus encore ceux ayant eu un parcours 8) ont choisi une exploitation dont les caractéristiques économiques sont sensiblement plus favorables (d'environ $+25 \%$ ) que celles d'agriculteurs de niveau d'études et de formation plus faible.

L'analyse conduite sur la base de variables mesurant d'une part la formation générale et d'autre part la formation professionnelle agricole ainsi que leur degré de continuité indique i) que les agriculteurs ont tendance à choisir des conditions économiques d'autant plus favorables qu'ils ont fait des études générales plus longues ; ii) l'existence d'une structure de complémentarité favorable entre formation générale et formation agricole initiale lorsque celle-ci s'applique à des individus ayant un niveau de formation générale relativement faible. Par ailleurs, on observe une structure de substitution difficile à gérer lorsque l'enseignement agricole et la formation technique s'appliquent à des jeunes de niveau d'études générales plus élevé. Dans ce cas, la formation agricole apporte à la formation générale une valeur ajoutée faible ou nulle.

\section{Dans quelle mesure les agriculteurs plus formés sont- ils plus efficaces pour rentabiliser leur exploitation?}

On observe qu'il existe une assez forte variabilité dans la capacité des agriculteurs à tirer profit de leur exploitation (compte tenu de ses caractéristiques techniques) ; de nouveau, on observe que la formation de l'agriculteur ne contribue que très faiblement $\left(R^{2}<0,04\right)$ à l'explication statistique de cette variabilité.

Cela dit, les analyses empiriques conduites montrent premièrement que la formation générale n'a pas d'impact positif sur la capacité de l'agriculteur à faire fonctionner son exploitation. Deuxièmement, elles montrent que la formation agricole initiale a globalement un impact très limité sur cette capacité ; mais il apparaît que l'effet tend à être davantage positif si la formation agricole s'appuie sur un niveau de formation générale suffisant. En troisième lieu, il apparaît que la formation continue, tout en offrant l'opportunité à des individus (dont certains sont en échec scolaire) d'acquérir une formation agricole, ne leur donne pas la possibilité d'obtenir des résultats économiques performants pour leur exploitation, et ce d'autant plus qu'il s'agit de stages de longue durée.

\section{A titre de conclusion}

Ces résultats de nature statistique appellent deux types de réflexions : la première est de l'ordre de leur compréhension "sensible", la seconde de celui de leur utilisation dans une perspective de politique de formation :

* il est simplement possible que la formation agricole spécifique ne soit pas un vecteur de transmission très efficace des compétences mobilisées par les agriculteurs et que celles-ci s'acquièrent en fait pour une large part sur le tas dans l'exercice même du métier. Il est possible aussi que l'encadrement agricole, les revues professionnelles et l'imitation inter-personnelle aient un impact positif substantiel, si bien que la variabilité de la formation individuelle se trouve gommée par l'influence de ces considérations environnementales.

* concernant la politique de formation, la première question un peu brutale serait évidemment de se demander comment on envisage de justifier qu'on dépense autant pour la préparation des exploitants agricoles dans la mesure où on obtient aussi peu des activités de formation engagées ; ce n'est bien sûr pas la bonne question. Il ne s'agit pas non plus directement de s'interroger sur la pertinence de l'exception agricole par rapport à l'éducation nationale, compte tenu du peu d'influence de la formation agricole et des effets plutôt positifs de la formation générale. Il est sans doute préférable de partir de l'idée que ces deux questions ne sont pas posées. Par contre dans le cadre des institutions existantes, une réflexion renouvelée sur une définition plus pertinente des formes et contenus de la formation professionnelle agricole, notamment continue, apparaît probablement incontournable au vu des résultats obtenus ici. Ce travail ne permet pas de préciser les contours de cette nouvelle politique de formation et ceux-ci doivent sans aucun doute être définis au sein des instances professionnelles de concertation. Il reste que les nouvelles dispositions qui seraient prises devraient, plus que dans le passé, faire l'objet d'une évaluation factuelle à l'aune non des opinions courantes mais des résultats obtenus.

\section{Pour en savoir plus : \\ "Evaluation de l'efficacité externe de la formation des agriculteurs", Stephan N. Thèse, $1996-300$ p. + ann. \\ "Evaluation de l'efficacité externe de la formation des agriculteurs", Mingat A., Stephan N. \\ Formation Emploi, N59, juill/sept. 1997}

\section{Rappel des dernières Notes}

96/6 Les taux de rendement sociaux "complets" de l'éducation : estimation à partir de la performance des pays en termes de croissance économique 96/7 La détermination progressive de l'enseignement de spécialité de la 3 ème à la Terminale

"Les Notes de l'Irédu" est une collection à parution irrégulière - Chaque numéro peut être obtenu contre 6 F en timbres auprès du service documentation de l'Irédu (Abonnement $50 \mathrm{~F}$ les 10 Notes) - ISSN en cours 
